\title{
Room-temperature preparation and characterization of poly (ethylene glycol)-coated silica nanoparticles for biomedical applications
}

\author{
Hao Xu, Fei Yan, Eric E. Monson, Raoul Kopelman* \\ Department of Chemistry, University of Michigan, Ann Arbor, Michigan 48109-1055
}

Received 1 April 2002; revised 4 November 2002; accepted 20 January 2003

\begin{abstract}
Monodisperse, spherical, polyethylene glycol (PEG)-coated silica nanoparticles have been prepared at room temperature and characterized for the purpose of biomedical applications. The particles were synthesized by the hydrolysis of tetramethyl orthosilicate (TMOS) in alcohol media under catalysis by ammonia, and their size can range from about 50-350 $\mathrm{nm}$ in diameter. We studied the particle size and size distribution using a scanning electron microscope (SEM) and an asymmetric field-flow fractionation (AFFF) multiangle static light-scattering instrument. The chemical and/or physical binding of PEG to the silica nanoparticles was studied by infrared spectroscopy, and the weight percentage of PEG attached to the particles was quantified. The PEG-coated silica nanoparticles showed enhanced colloidal stability when redispersed into aqueous solutions from the dried state as a result of the steric stabi-
\end{abstract}

lization function of the PEG polymer grafted on the surface of particles. A nonspecific protein-binding test was also carried out to show that the PEG coating can help reduce the protein adsorption onto the surface of the particles, relating to the biocompatibility of these PEG-coated particles. Also, the inclusion of magnetic nanoparticles into the silica particles was shown as an example of the possible applications of PEG-coated silica particles. These silica nanoparticles, as a matrix for encapsulation of certain reagents, have potential for applications to in vivo diagnosis, analysis, and measurements inside intact biologic systems. (C) 2003 Wiley Periodicals, Inc. J Biomed Mater Res 66A: 870-879, 2003

Key words: room-temperature preparation; poly(ethylene glycol); silica nanoparticles; biocompatible PEG coating; biomedical applications

\section{INTRODUCTION}

Silica nanoparticles, produced by the sol-gel process that uses silicon alkoxides as starting materials, have received much attention for many years because of their practical importance in a variety of fields. ${ }^{1-12}$ In recent years, the newly emerging area of in vitro and in vivo diagnosis, analysis, and measurements inside intact biologic systems (e.g., tissues, blood, and single cells), with the use of nanoparticle devices, has attracted considerable attention. . $^{2,5,8,13-17}$ Some of the typical applications in this area may include (1) dyedoped nanospheres as nanosensors for intracellular measurements ${ }^{14-16}$; (2) magnetic nanoparticles for diagnostic magnetic resonance imaging ${ }^{8,13}$; and (3)

Correspondence to: Raoul Kopelman; e-mail: kopelman@ umich.edu

Contract grant sponsor: NIH; contract grant number: 8 RO1 EB00250 09

Contract grant sponsor: NCI; contract grant number: N01CO-07013

(c) 2003 Wiley Periodicals, Inc. nanospheres coated with biodegradable materials as injectable blood-persistent systems for the controlled release of drugs, site-specific drug delivery, and medical imaging. 8,17

Our main focus in this work is to develop a method for producing "biofriendly," spherical, and monodisperse silica nanoparticles, prepared by the sol-gel method, for use in the above newly emerging research area. We chose to use silica as a matrix in our work here because it has been one of the most commonly used matrices for making nanoparticles for in vivo applications, and it has several superior properties. Silicate glass is a porous, high-purity, optically transparent and homogeneous material, ${ }^{18}$ thus making it an ideal choice as a matrix for the encapsulation of optically active reagents. Also, the preparation of silica particles is technically simple, and tailoring their physicochemical properties (i.e., pore size or innersurface hydrophobicity) can be achieved easily by varying the processing conditions and the concentration or type of reactants used. Furthermore, the silica matrix makes it possible to retain, to a much larger extent compared to organic polymers, the specificity 
and reactivity of biologic molecules (i.e., proteins) in the solid state and provides morphologic and structural control that is not available when the biologic molecules are simply dissolved in aqueous media. ${ }^{19}$

The first successful method for the preparation of monodisperse, spherical silica nanoparticles was reported by Stöber et al. in $1968,{ }^{20}$ based on the hydrolysis of tetraethyl orthosilicate (TEOS) in alcohol media under catalysis by ammonia. With their procedure, it is possible to obtain different-sized silica spheres in the whole colloidal size range, simply by changing the initial concentrations of the reagents.

In our early experimental attempts to produce silica nanoparticles, we sometimes found that, after collecting the particles as a dry solid, aggregation among particles took place when we resuspended them in an aqueous solution for applications, because the suspended silica particles formed agglomerates, probably due to (interparticle) hydrogen bonding. ${ }^{5}$ Steric hindrances, by the use of polymers (steric stabilizers) adsorbed or grafted onto particle surfaces, have been one answer to the problems posed by aggregations among particles in an aqueous suspension. In this case, direct contacts among particles are prevented by the polymer chains extending into the medium. Therefore, no aggregates of particles will be formed, or the rate of coagulation will be decreased, depending on the degree of coverage of polymers on the particles and the thickness of the polymer layer. ${ }^{21-23}$ We chose to use, as a coating steric stabilizer, a polyethylene glycol (PEG) form that is covalently bound to the particle at one end but extends far into the surrounding medium. Thus, in an aqueous suspension, the aggregation among the nanoparticles is prevented by the repulsion force and solvation layer of the PEG surface moiety. ${ }^{24}$ Some other important advantages of having PEG coatings on the silica nanoparticles concern their applications inside biologic samples. First, PEG is nontoxic, and its attachment to silica nanoparticles provides a biocompatible and protective surface. ${ }^{25}$ Also, the PEG coatings reduce protein and cell adsorption onto the particles and can reduce the rate of clearance (through organs such as the kidney) of "PEGylated" materials, thus increasing the particle circulation time for in vivo applications. ${ }^{26-28}$

Here, we describe a method for the preparation of PEG-coated silica nanoparticles, using a sol-gel process, for biomedical applications. The effects of the reactant and catalyst concentrations on the size distribution of the silica particles are studied by scanning electron microscopy (SEM) and asymmetric field-flow fractionation (AFFF) multiangle static light-scattering experiments. We provide evidence of the PEG coating with Fourier transform infrared (FT-IR) studies and also determine the weight percentage of PEG in the particles. The inclusion of magnetic nanoparticles into the silica matrix is demonstrated and discussed briefly, indicating one of the possible applications of PEG-coated silica particles. Also given are nonspecific protein-binding studies that relate to the biocompatibility of the PEG-coated silica nanoparticles.

\section{MATERIALS AND METHODS}

\section{Materials}

Reagents

All reagents and solvents were purchased from SigmaAldrich (Milwaukee, WI) unless otherwise noted. Ethanol (200 proof) was obtained from Pharmco Products, Inc. (Brookfield, CT). Texas Red-labeled bovine serum albumin (BSA) used in the nonspecific protein-binding test was obtained from Molecular Probes (Eugene, OR).

\section{Preparation of PEG-coated silica nanoparticles}

The processing steps for PEG-coated silica nanoparticles are as follows: PEG polymers of different molecular weights were dissolved in mixed solution of ammonium hydroxide and methanol. Upon mixing, the solution became transparent, and tetramethyl orthosilicate (TMOS) was added dropwise to initiate the hydrolysis reaction. The solution was then stirred vigorously at room temperature for a few hours before the reaction was stopped. A typical reaction solution, which gives an average particle size of $\sim 100 \mathrm{~nm}$, consisted of PEG molecular weight (MW) 5000 monomethyl ether (2 g), methanol (99.9\%, $24 \mathrm{~mL}$ ), ammonium hydroxide (30 wt \% ammonia, $6 \mathrm{~mL})$, and TMOS $(99.9 \%, 0.2 \mathrm{~mL})$.

After we stopped the reaction, we then added a liberal amount of ethanol to the reaction solution and transferred the mixture to an Amicon ultrafiltration cell (Millipore Corp., Bedford, MA). A $100-\mathrm{kDa}$ membrane was used to separate the silica particles from the unreacted monomers, PEG, and ammonia, under a pressure of 10 psi. The particles were further rinsed with at least $500 \mathrm{~mL}$ distilled water and $200 \mathrm{~mL}$ ethanol to ensure that all unreacted PEG and TMOS had been removed from the silica particles. The silica particle suspension was then passed through a suction filtration system (Fisher, Pittsburgh, PA) with a $0.02-\mu \mathrm{m}$ filter membrane to collect the particles, which were then dried to yield a final product of PEGylated silica nanoparticles.

\section{Experimental methods}

\section{SEM imaging}

To prepare the samples for SEM studies, dried silica nanoparticles were dispersed in water, and the resultant suspensions were sonicated for 1-2 $\mathrm{h}$. We then placed a drop of the silica particle suspension on a piece of microglass slide 
(Arthur H. Thomas Co., Philadelphia, PA) attached to a metal grid coated with carbon film, and dried it gradually at room temperature. The sample was then sputter coated with gold and visualized with a Philips XL30FEG SEM to assess the particle size and shape.

AFFF multiangle static light-scattering measurements

Mean particle size, size distribution, and polydispersity of the silica nanoparticles (dried, then redispersed in aqueous buffer solutions) were measured at room temperature by a Dawn EOS Light-Scattering Instrument (Wyatt Technology Corp., Santa Barbara, CA).

Particles were first suspended from powder at a concentration of $2 \mathrm{mg} / \mathrm{mL}$ in a running buffer [400 ppm sodium dodecylsulfate (SDS), 200 ppm sodium azide, and 100 ppm $\mathrm{NaCl}$ in ultrapure water filtered to $0.1 \mu \mathrm{m}$ ] and sonicated for $30 \mathrm{~min}$ to $1 \mathrm{~h}$ to reduce aggregation. Samples were filtered through a $1.6 \mu \mathrm{m}$ glass-fiber syringe filter (13-mm diameter, GF/A grade; Whatman) before injection into the $20-\mu \mathrm{L}$ sample loop of an AFFF liquid separation system (Consenxus, Germany). The output of the separation channel was directed into the multiangle static light-scattering instrument, where 15 detectors simultaneously measured the scattered intensity every $0.5 \mathrm{~s}$. The multiangle data were used at each time point to calculate the particle number density and volume within that sample slice (assuming validity of the Raleigh-Gans-Debye approximation; i.e., spherical scatterers with an index of refraction close to that of the aqueous phase). We then accumulated this information into a histogram of differential mass fraction versus hydrodynamic radius for all identified sample peaks, without making any assumptions about the distribution of sizes present in the sample.

\section{FT-IR study}

Evidence of the PEG coating was given by FT-IR spectra of the dried silica particles, recorded by a Perkin Elmer Spectrum BX FT-IR system (Perkin-Elmer Limited, Beaconsfield Bucks, UK). We first prepared the samples by mixing the pure PEG polymers or silica nanoparticles with $\mathrm{KBr}$ by grinding, then placed some of the powder mixture into a metal Econo mount (Mason Box Co., Attlebord Fall, MA) to form a pellet in the mount by applying pressure to both ends. The mount was then placed into the FT-IR system, with the sample pellet in it, for analysis.

\section{Nonspecific protein-binding tests}

The approach we used here to probe the protein adsorption to various formulations of the silica nanoparticles was modified from a method used for thin films. ${ }^{29}$ We dissolved $5 \mathrm{mg}$ of Texas Red-labeled BSA in $1 \mathrm{~mL}$ of Dulbecco's phosphate-buffered saline (DPBS, pH 7.4), which was then stored at $-20^{\circ} \mathrm{C}$. For the binding experiments, we suspended each sample at $0.67 \mathrm{mg} / \mathrm{mL}$ in $3 \mathrm{~mL}$ of DPBS and sonicated it for $1 \mathrm{~h}$. We added $2 \mu \mathrm{L}$ of the Texas Red-labeled BSA solution to each sample and incubated the samples at $37^{\circ} \mathrm{C}$ for $2 \mathrm{~h}$. Then, we centrifuged the samples for $15 \mathrm{~min}$ at 8000 rpm using a Marathon 21000 1L Ventilated Centrifuge (Fisher Scientific, PA). Here, we chose carefully the rate used for the centrifugation process to make sure that all the silica particles (part of which were particles with protein bound to them) are separated from the bulk solution at the bottom, but the unbound dye-labeled proteins should still remain in the solution. After centrifugation, the supernatant was removed, and the precipitates were rinsed three times with DPBS buffer and resuspended in $3 \mathrm{~mL}$ of buffer solution before measurements. We performed the protein adsorption measurement by looking directly at the fluorescence of the particle suspensions using a FluoroMax-2 Spectrofluorometer (ISA Jobin Yvon Spex, Edison, NJ). All FluoroMax-2 functions were under the total control of DataMax spectroscopy software, and all measurements were taken at room temperature. A magnetic stirrer was used to generate a more uniform suspension during the measurements. The fluorescence intensity was measured at $610 \mathrm{~nm}$, with an excitation wavelength of $590 \mathrm{~nm}$.

Preparation and characterization of magnetic silica nanoparticles

The magnetic silica nanoparticles were prepared in the same manner as the PEG-coated silica nanoparticles, except that $10 \mathrm{mg}$ of a fluorescent dye $\left[\mathrm{Ru}(\mathrm{dpp})_{3}\right] \mathrm{Cl}_{2}$ and $5 \mathrm{mg}$ $\gamma-\mathrm{Fe}_{2} \mathrm{O}_{3}$ were added to the reaction mixture, before the addition of TMOS. To confirm the incorporation of the magnetic components, we designed an experiment involving fluorescence imaging, in which the excitation source was a mercury lamp (Olympus, Melville, NY) attached to an Olympus inverted fluorescence microscope, and the detector was a liquid nitrogen-cooled charge-coupled device (CCD) purchased from Princeton Instruments. Suspensions of iron oxide-and ruthenium dye-doped silica nanoparticles were placed in a rectangular capillary $(100 \mu \mathrm{m} \times 2 \mathrm{~mm} \times 5 \mathrm{~cm})$, which was then placed on a microscope slide and sealed with wax. A piece of iron wire passing through a solenoid (a roll of coiled copper) was held above the capillary by a micropositioner. When current is applied to the solenoid, the iron magnetizes, and any magnetic particles near it are pulled or form chains that rush toward the magnet.

\section{RESULTS AND DISCUSSION}

\section{Formation of silica nanoparticles}

In our method of producing monodisperse and spherical PEGylated silica nanoparticles, PEG and TMOS proceed through the following steps to form the final particles:

$$
\begin{aligned}
\cdots \mathrm{Si}-\mathrm{OR}+\mathrm{HO}\left(\mathrm{CH}_{2} \mathrm{CH}_{2} \mathrm{O}\right)_{\mathrm{n}} \mathrm{H} \leftrightarrow \\
\cdots \mathrm{Si}-\mathrm{O}\left(\mathrm{CH}_{2} \mathrm{CH}_{2} \mathrm{O}\right)_{\mathrm{n}} \mathrm{H}+\mathrm{ROH}
\end{aligned}
$$


TABLE I

Particle Size Data

\begin{tabular}{|c|c|c|}
\hline Sample & Reaction Composition & $\begin{array}{l}\text { Particle Size }(\mathrm{nm}) \\
\text { Determined by SEM }\end{array}$ \\
\hline Sample 1 & $3 \mathrm{~mL} \mathrm{NH}_{3} \cdot \mathrm{H}_{2} \mathrm{O}, 24 \mathrm{~mL} \mathrm{MeOH}, 0.1 \mathrm{~mL}$ TMOS, and $1 \mathrm{~g}$ PEG monomethyl ether MW 5000 & $55.5 \pm 13.8$ \\
\hline Sample 2 & $3 \mathrm{~mL} \mathrm{NH} \cdot \mathrm{H}_{2} \mathrm{O}, 12 \mathrm{~mL} \mathrm{MeOH}, 0.1 \mathrm{~mL}$ TMOS, and $1 \mathrm{~g}$ PEG monomethyl ether MW 5000 & $118.8 \pm 18.6$ \\
\hline Sample 3 & $5 \mathrm{~mL} \mathrm{NH} \cdot \cdot \mathrm{H}_{2} \mathrm{O}, 12 \mathrm{~mL} \mathrm{MeOH}, 0.2 \mathrm{~mL}$ TMOS, and $1 \mathrm{~g}$ PEG monomethyl ether MW 5000 & $238.1 \pm 26.8$ \\
\hline Sample 4 & $7.5 \mathrm{~mL} \mathrm{NH}{ }_{3} \cdot \mathrm{H}_{2} \mathrm{O}, 12 \mathrm{~mL} \mathrm{MeOH}, 0.2 \mathrm{~mL}$ TMOS, and $1 \mathrm{~g}$ PEG monomethyl ether MW 5000 & $328.5 \pm 35.7$ \\
\hline
\end{tabular}

$$
\begin{gathered}
\cdots \mathrm{Si}-\mathrm{OR}+\mathrm{H}_{2} \mathrm{O} \leftrightarrow \cdot \mathrm{Si}-\mathrm{OH}+\mathrm{ROH} \\
\cdots \mathrm{Si}-\mathrm{OR}+\mathrm{HO}-\mathrm{Si} \leftrightarrow \\
\cdots \mathrm{Si}-\mathrm{O}-\mathrm{Si}+\mathrm{ROH}
\end{gathered}
$$

The transesterification reaction between TMOS and PEG [Eq. (1)] competes with hydroxylation [Eq. (2)]. The equilibrium, Eq. (2), is followed by the condensation step [Eq. (3)], thus leading to the formation of a silica network. However, Eqs. (2) and (3) are predominant over Eq. (1). ${ }^{30}$

As observed by Stöber et al., in general, smaller particles and narrower size distributions are obtained with smaller alcohols as solvent; faster reactions and smaller particles are obtained with smaller alkoxides. ${ }^{20}$ Usually, small physical size of particles is desired for in vivo studies, because smaller particles may cause less perturbation (compared to the same kind of particles with larger size) to the biologic system when inserted, so we used the smallest alkoxide (TMOS) and alcohol $(\mathrm{MeOH})$ in our method for this purpose. Another important factor in controlling the size and size distribution of the silica nanoparticles is the concentration of the precursor TMOS. Van Helden et al. reported that the particle size and the width of the size distribution increase, and also the sphericity of the particles deteriorates, when the concentration of precursor (TEOS used in their work) exceeds about 0.2 $M{ }^{7}$ So, the TMOS concentrations used in all the experiments described here were kept below this concentration, to obtain spherical particles with uniform sizes.

A typical SEM image of PEGylated silica nanoparticles (Sample 2 in Table I) is shown in Figure 1. As can be seen, the particles are spherical and the polydispersity is small. It should again be noted that we did not take the SEM samples directly from the reaction solution but prepared them by redispersing dried silica particles in distilled water. When necessary, PEGcoated silica nanoparticles of different sizes can be prepared by changing the synthetic conditions; some examples are shown in Table I. The mean diameters and standard deviations of these silica particles are determined from the SEM results. As can be seen in Table I, by changing the composition of the reaction solution, particles ranging from about $50-350 \mathrm{~nm}$ can be prepared. In these samples, the main parameters

we changed, to control the particle sizes, were the initial concentrations of ammonia and water (i.e., the amount of ammonium hydroxide added with respect to the amount of alcohol). The trend observed here is that, from Samples 1-4, the increase in the amount of $\mathrm{NH}_{3} \cdot \mathrm{H}_{2} \mathrm{O}$ resulted in an increase in the particle size. This is consistent with results obtained by Stöber et al., ${ }^{20}$ who have shown that in alcohol/water/ammonia systems, particles prepared from TEOS increased in size with an increase in catalyst concentration. In our experiments, different TMOS concentrations had no significant influence on the particle sizes, because they were all kept well below $0.2 \mathrm{M}$. The amount of PEG monomethyl ether MW 5000 used in the synthesis of these samples was kept constant, because the effect of the amount of PEG used was not found to be significant.

\section{Colloidal stability of the PEGylated silica nanoparticle suspensions}

Figure 2 demonstrates a typical AFFF multiangle static light-scattering result of the hydrodynamic radius of particles (or clusters) in Sample 2. It can be

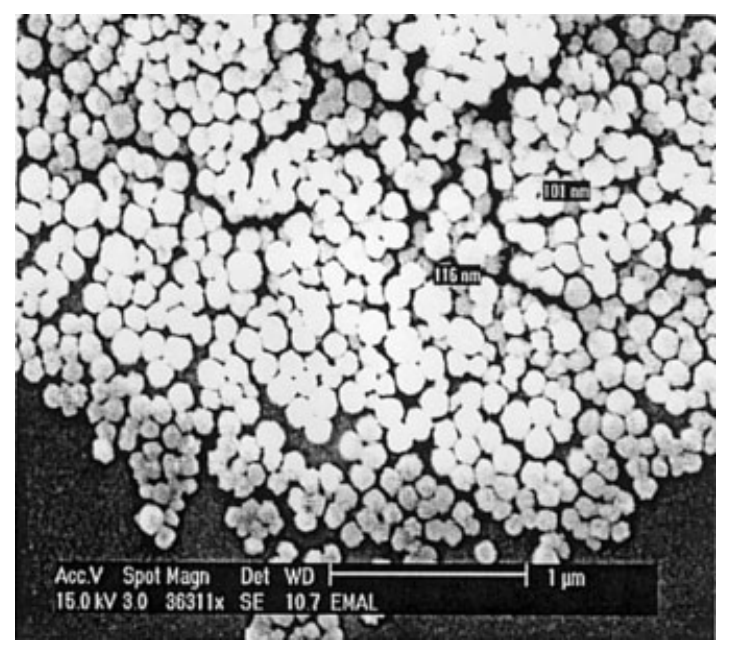

Figure 1. A typical SEM image of PEGylated silica nanoparticles. The scale bar is $1 \mu \mathrm{m}$. The particles were prepared with PEG MW 5000 monomethyl ether (2 g), methanol $(99.9 \%, 24 \mathrm{~mL})$, ammonium hydroxide (30 wt \% ammonia, 6 $\mathrm{mL})$, and TMOS $(99.9 \%, 0.2 \mathrm{~mL})$. 


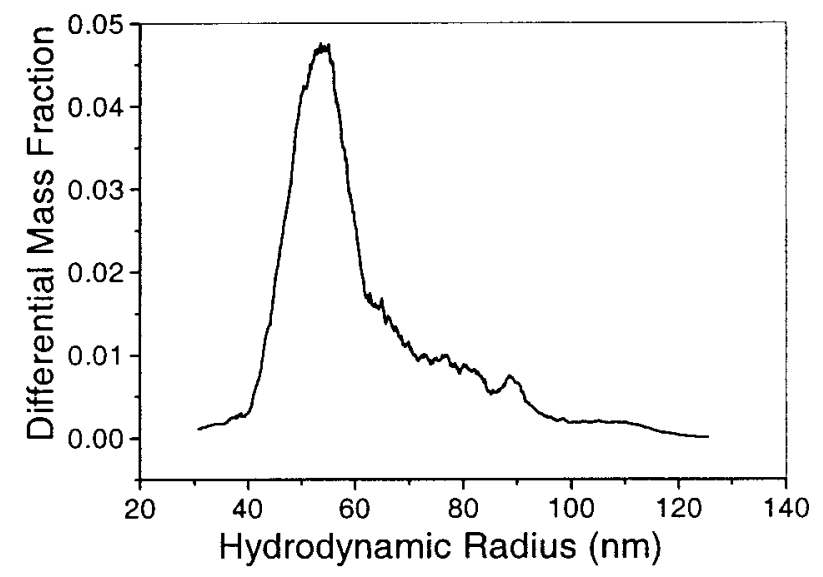

Figure 2. AFFF multiangle static light-scattering result, with the size distribution of the same batch of silica nanoparticles as depicted in Figure 1.

seen that the average size of the particles was about $110 \mathrm{~nm}$ in diameter, in agreement with the corresponding SEM result in Table I. From the closeness of this value to the SEM result, it could be inferred that there is little aggregation when the PEG-coated silica nanoparticles are suspended in a buffer solution during the light-scattering experiments. This property is crucial for the in vivo applications of silica nanoparticles, in that they need to be well separated in the suspension, so that they do not cause much perturbation (related to the size of particles) when applied inside biologic systems. For example, aggregates of particles may cause stronger perturbations in biologic systems once injected (e.g., aggregates of particles may clog the artery and cause animal death during in vivo toxicology studies), simply because they are larger in comparison to well-separated individual nanoparticles. This problem is alleviated by the steric stabilization function of the PEG 5000 monomethyl ether coatings on the particles, as demonstrated in Figures 1 and 2.

It is known that, in an aqueous dispersion, the electrostatic stabilization of the noncoated silica dispersion will be eliminated when there is high ionic strength. ${ }^{21}$ The electrostatic repulsion then only has a very short range and no longer protects the silica particles against coagulation. As mentioned earlier, in both polar and apolar solvents, the colloidal particles can be stabilized sterically by the presence of polymers adsorbed on or chemically bound to the surface of the colloidal particles. So when the electrostatic stabilization of the silica dispersion is eliminated by the high ionic strength, the polymer layer on the particle surface will prevent the coagulation of the particles or, when the layer is not thick enough, may reduce the coagulation rate. ${ }^{21}$ We found in our experiments that the effective binding of PEG resulted in an increase in the stability of the silica nanoparticle suspension against the addition of electrolyte. In this experiment, we prepared $2 \mathrm{mg} / \mathrm{mL}$ suspensions of PEG 5000 monomethyl ether-coated silica nanoparticles and pure silica nanoparticles (made using the same recipe) in distilled water under the same conditions, and sonicated them for $1 \mathrm{~h}$. After we added an $\mathrm{NaCl}$ solution to both samples to reach a final salt concentration of $\sim 0.15 \mathrm{M}$ (close to physiologic conditions), the noncoated nanoparticle suspension became turbid immediately and lost flow birefringence. All particles settled right away and would not resuspend after gentle shaking (i.e., large aggregates of particles could be seen in the solution, and the fine suspension before the addition of $\mathrm{NaCl}$ could not be recovered). In contrast, the PEG-coated nanoparticle suspension was more stable under this condition, still showing flow birefringence after the addition of $\mathrm{NaCl}$ without becoming turbid right away. These silica particles in the salt solution did not aggregate and settle during a time period of $\sim 10-30 \mathrm{~min}$. We found, however, that the particles did settle gradually after this period of time, but that by gentle shaking they could be resuspended to form a suspension similar in appearance to that before the addition of salt. These results indicate that the PEG coatings provide a steric hindrance between the suspended silica particles when there is high ionic strength, thus, at least to a large extent, helping to decrease the rate of coagulation of particles under this condition. It should be noted here that we found, under higher $\mathrm{NaCl}$ concentrations (higher ionic strength; i.e., 0.2-0.5 M), the suspended PEG-coated particles became less stable compared to the previously described particles. This implies that, at this stage, the degree of coverage or the thickness of the polymer layer on the particle surface may not be high enough to stabilize the particles at very high ionic strength. Thus, further improvements to increase the degree of coverage of PEG polymers or the thickness of the polymer layer might be necessary to achieve even better colloidal stability of PEGylated silica nanoparticle suspensions.

\section{FT-IR measurements}

The chemical binding of PEG to the silica nanoparticles was suggested by infrared spectroscopy, as demonstrated in Figure 3. The spectrum of the pure PEG MW 5000 monomethyl ether [Fig. 3(b)] showed many characteristic peaks at $843,960,1107,1146,1240,1280$, $1346,1466,2886$, and $2960 \mathrm{~cm}^{-1}$. Some of the reported strong absorptions of PEG are assigned to the $-\mathrm{CH}_{2} \mathrm{CH}_{2}$-stretching around 2886 and $2960 \mathrm{~cm}^{-1}, 29,31$ which demonstrates the presence of saturated carbons $-\left(\mathrm{CH}_{2} \mathrm{CH}_{2}\right)_{n}$-. Figure 3(a) shows the FT-IR spectrum of silica nanoparticles without the addition of PEG dur- 

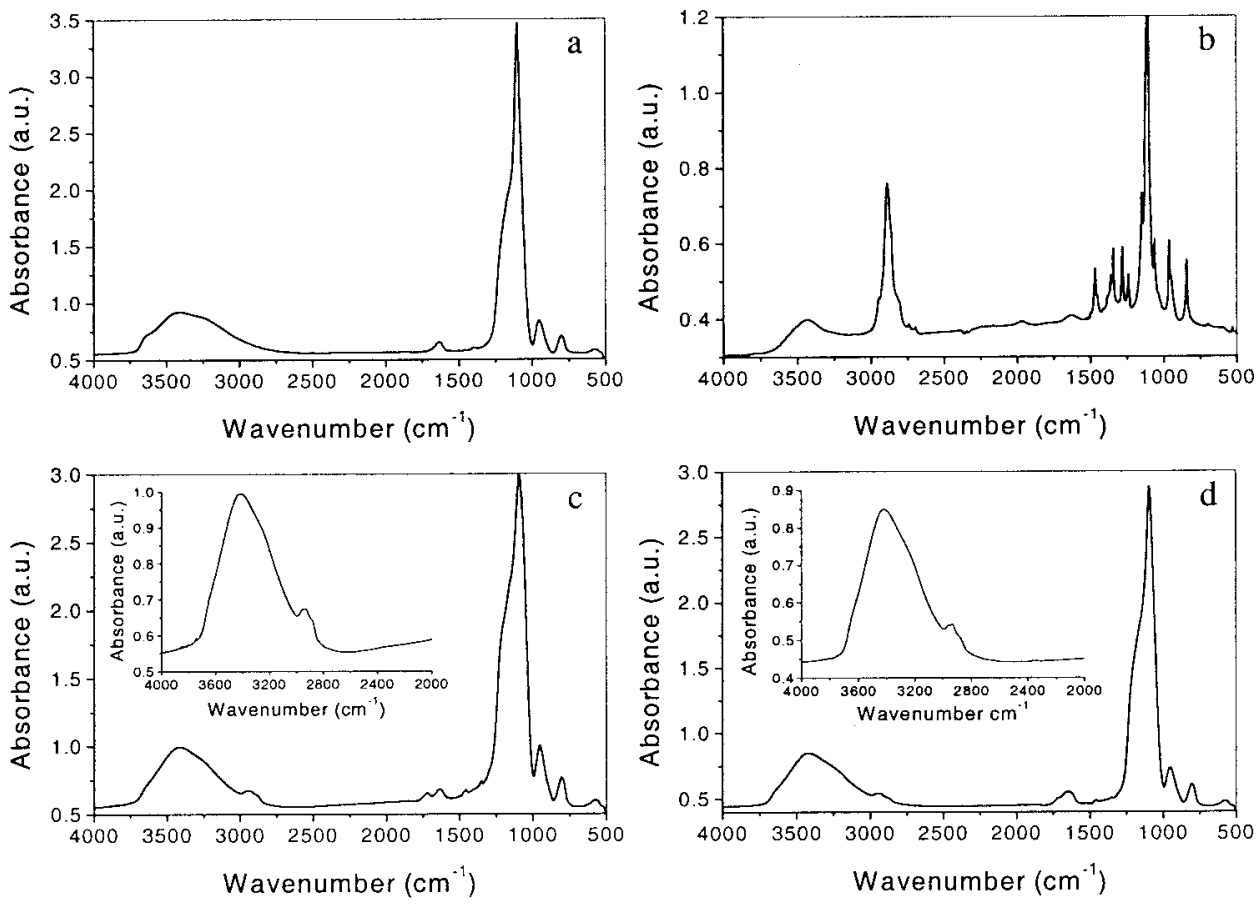

$y=102.1 x+16.3 \quad R^{2}=0.9983$

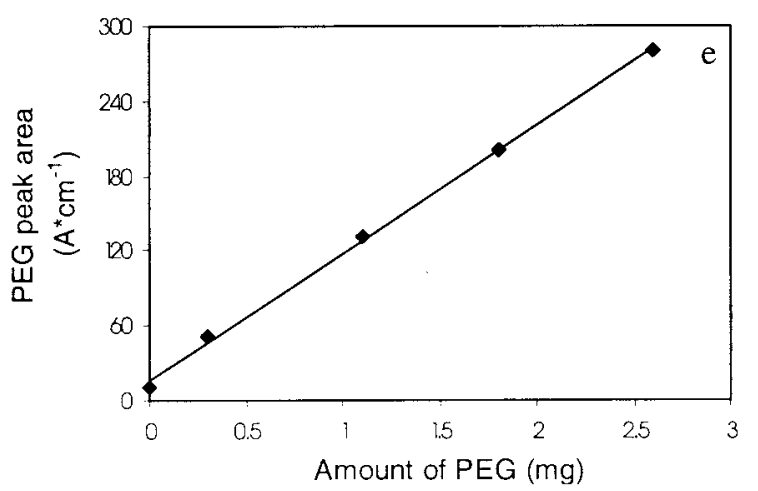

Figure 3. FT-IR spectra of (a) pure silica nanoparticles, (b) pure PEG MW 5000 monomethyl ether, (c) PEGylated silica nanoparticles in which PEG is added simultaneously with other reactants, (d) PEGylated silica nanoparticles in which PEG is added after $2 \mathrm{~h}$, and (e) calibration curve for pure PEG MW 5000 monomethyl ether in a $\mathrm{KBr}$ pellet.

ing synthesis. As can be seen, pure silica particles possess characteristic peaks at 796, 950, 1065, 1400, 1633 , and $3400 \mathrm{~cm}^{-1}$. The very broad peak in the region between 3100 and $3600 \mathrm{~cm}^{-1}$ indicates the presence of exchangeable protons, in this case, mostly from $\mathrm{OH}$ groups on the surface of the particles. The spectrum of PEGylated silica nanoparticles [Fig. 3(c)] gave new absorption peaks at 2886 and $2960 \mathrm{~cm}^{-1}$, corresponding to some of the characteristic peaks of PEG MW 5000 monomethyl ether. So these peaks from the PEG-doped silica particles (exactly the same place as the characteristic peaks in pure PEG) should be from the PEG content, because these peaks were not present in the pure silica particle sample, and the only difference between Figure 3( $a$ and $c$ ) is the addition of
PEG. These peaks suggest the chemical binding of PEG to the silica particles, though the change is obscured by the overlapping peaks of the pure silica nanoparticles. It should be noted here that the PEGcoated silica particles were washed with large amounts of ethanol and water before measurements, so presumably the PEG polymers that are only physically adsorbed on the surface of the particles would be rinsed off, and the signal was only from chemically attached PEG polymers. Figure 3(d) shows another FT-IR spectrum of PEGylated silica particles, in which the only difference compared to Figure 3(c) is that the PEG MW 5000 monomethyl ether was added after the particles were formed (i.e., $2 \mathrm{~h}$ after reaction started). Our original purpose in this experiment was to see 
whether PEG could be bound to particles that have already been formed, and we found that PEG polymers were still able to bind to the silica particles in this case, as evidenced by the presence of the characteristic peaks of PEG 5000 monomethyl ether. In this case, as shown earlier in Eqs. (1) through (3), the PEG polymer still attached to the particle surface as a result of the reaction between the silanol groups on the particle surface and the end alcohol groups on the PEG chain, which is a simple condensation reaction that produces an ester bond $(\mathrm{Si}-\mathrm{O}-\mathrm{C}){ }^{29}$

The percentage of PEG content in the silica nanoparticles was quantified based on the FT-IR spectra. As shown in Figure 3(e), we obtained a calibration curve for pure PEG MW 5000 monomethyl ether using the $\mathrm{KBr}$ pellet method, in which the same amount of $\mathrm{KBr}$ (i.e., $120 \mathrm{mg}$ ) and varied amounts of PEG MW 5000 monomethyl ether (i.e., 0.3, 1.1, 1.8, and $2.6 \mathrm{mg}$ ) were used. According to the calibration equation, about 0.04 and $0.02 \mathrm{mg}$ PEG 5000 monomethyl ether were incorporated into the $2 \mathrm{mg}$ of PEGylated silica nanoparticles in Figure 3(c and d). Therefore, the PEG content for these two specific batches of silica nanoparticles is about $2 \%(\mathrm{w} / \mathrm{w})$, and $1 \%(\mathrm{w} / \mathrm{w})$, respectively. It should be noted here that, in our method, we did not use the optimum conditions for the condensation reaction between the silanol groups on the particles and the end alcohol groups on the PEG chain. It was reported that this reaction would be favored by acid catalysis and heating of the reaction solution, 8,29 and a high degree of PEG coverage would result. However, we chose not to use these conditions to increase the PEG coverage on the particles for the following reasons: First, the formation of silica nanoparticles with use of the Stöber method requires base catalysis; second, the in vivo applications of these PEGylated silica particles inside intact biologic systems always involve the encapsulation of certain biologic molecules (i.e., proteins) and organic dyes into the particles, and often these reagents would be sensitive to high temperature. So the synthesis at room temperature provides a milder environment and makes the encapsulation of these reagents possible; thus, a larger variety of reagents can be used for applications. Though we did not use the above optimum conditions in our method, a higher degree of PEG coverage may be achieved by a longer reaction time.

The potential advantage of the method used in Figure 3(d) is that one can choose to add PEG after the particles are already formed, so that, presumably, all the PEG sidechains are on the outside of the particles and extend farther into the medium, possibly resulting in thicker polymer layers on particle surfaces. Thus, they might work more efficiently as a steric stabilizer and, at the same time, would not interfere with the interior structure of the particles. This will be tested in future work.

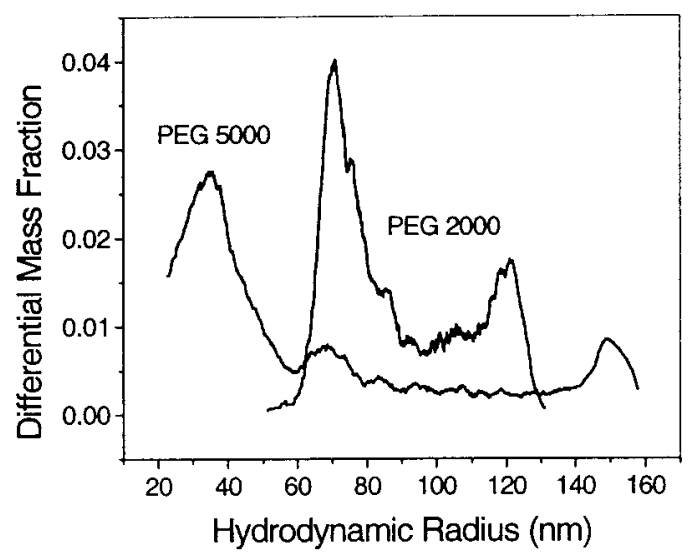

Figure 4. AFFF multiangle static light-scattering results for PEGylated silica nanoparticles with the use of PEG 2000 and PEG 5000 monomethyl ether. The particles were prepared with PEG MW 5000 monomethyl ether (2 g) or PEG MW 2000 monomethyl ether (2 g), methanol (99.9\%, $48 \mathrm{~mL})$, ammonium hydroxide (30 $\mathrm{wt} \%$ ammonia, $6 \mathrm{~mL}$ ), and TMOS $(99.9 \%, 0.2 \mathrm{~mL})$.

\section{Effects of different PEG molecular weights}

The effect of the molecular weight of PEG monomethyl ether on the colloidal stability of silica nanoparticle suspensions was also studied by AFFF multiangle static light-scattering measurements, and the results are shown in Figure 4. As can be seen, for two batches of particles prepared with the same recipe as in Sample 1, particles with the PEG 5000 coating are shown to be smaller in size when suspended in the buffer used for the light-scattering experiments, indicated by the peak on the left. In contrast, as shown in Figure 4, the PEG 2000 curve is more on the right, meaning that these particles should be larger compared to the ones made with the use of PEG 5000. However, according to the corresponding SEM micrographs, the PEG 5000-coated silica particles are similar in size to the PEG 2000-coated ones. So we assume that the difference between the size distribution of these two kinds of particles shown in the lightscattering result was possibly due to the formation of aggregates of the PEG 2000-coated particles during the light-scattering experiments, resulting from the different steric stabilization effects of PEG 5000 and PEG 2000 polymers on colloidal suspensions. PEG 5000 has longer sidechains, extending farther into the medium to form a thicker layer on the particle surface, compared to PEG 2000, so it functions more effectively as a steric stabilizer. Thus, presumably, there should be little aggregation among particles during the lightscattering measurements when dried particles are dispersed in aqueous buffers. On the other hand, when PEG 2000 was used, aggregation was more of a problem, because it is not as effective a steric stabilizer as PEG 5000 monomethyl ether; thus, larger and less 


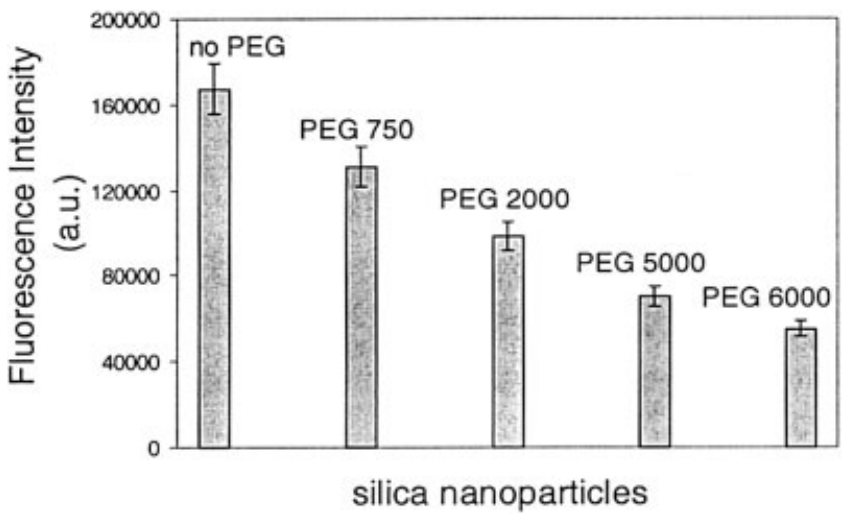

Figure 5. Protein adsorption on bare and PEGylated silica nanoparticles, indicated by the fluorescence intensities of the dye-labeled BSA adsorbed on particle surfaces; the error bars represent the standard deviation of three measurements.

uniform sizes (possibly resulting from clusters of individual particles) were observed.

\section{Nonspecific protein-binding tests}

Protein adsorption plays a major role in a variety of important biologic-related processes. Biocompatible materials are required to eliminate, or largely reduce, the adsorption of blood proteins (e.g., to avoid surface-induced thrombosis). The term "biocompatible" is used to define the property of materials introduced in the human or animal body without causing any allergic or rejective reactions. PEG polymers are now one of the most popular materials to modify particulate surfaces to avoid recognition by cells of the mononuclear phagocyte system (MPS). PEG is unusually effective at excluding other polymers from its presence in aqueous solution. This property is thought to be directly related to its ability to repel proteins. ${ }^{32}$ To produce PEGylated surfaces, it is necessary to form a permanent chemical bond between the substrate and PEG. If PEG is only physically adsorbed, it will eventually be removed by biofluids, because it is soluble in both water and a great variety of organic solvents. ${ }^{26}$

Our goal in this experiment was to determine the optimal PEG coatings on silica nanoparticles, to reduce nonspecific protein adsorption on the particles. We prepared silica nanoparticles using the same recipe as in Sample 2 in Table I, in which the MW of PEG was varied from 750 to 6000 . The protein (labeled with Texas Red) bound to the particles was analyzed with a spectrofluorometer.

Figure 5 shows that the total amount of adsorbed BSA detected on the surface of silica nanoparticles varied with different PEG polymer chain lengths. As can be seen, when the MW of the PEG coating on the silica nanoparticles increased from 750 to 6000 , the BSA adsorption on the particles was reduced remarkably compared to that of the bare silica nanoparticles (i.e., the amount of adsorbed protein on the PEG 6000 coated particles was $\sim 3$ times less than that on the bare silica particles). We found this trend quite repeatable (by looking at both the particles with bound proteins and the supernatant containing the unbound proteins) and consistent with the findings reported previously (i.e., the protein adsorption is a function of chain length): The longer the polymer, the more effective its prevention of protein adsorption. ${ }^{33}$ This property of the PEG-coated silica nanoparticles will be a critical advantage in future in vivo studies.

\section{Preliminary results on magnetic nanoparticle inclusion}

The inclusion of magnetic components in the silica matrix is demonstrated here to provide an example of the potential applications of the PEGcoated silica nanoparticles. The main application for this kind of magnetic nanoparticle device is to increase the contrast in diagnostic magnetic resonance imaging. By adding iron oxide nanoparticles to a typical silica particle reaction solution, we were able to make silica nanoparticles doped with magnetic components. A fluorescent dye, $\left[\mathrm{Ru}(\mathrm{dpp})_{3}\right] \mathrm{Cl}_{2}$, was also incorporated into the silica matrix, together with the magnetic components, and its fluorescence was used to help determine whether the magnetic components were encapsulated inside the silica particles. Figure 6(a) shows the bright-field image of the silica nanoparticles, prepared by the above method, when an iron wire magnet was centered in the image, and Figure 6(b) shows one stage of the motion of the silica nanoparticles (toward the magnet) when the iron wire was positioned in the right corner of the image. We performed a similar experiment on the same particles under the illumination of a mercury lamp, where the motion of the fluorescence signals toward the magnet could be observed [Fig. 6(c and d)]. We believe that ruthenium dye molecules attached to the outside of the particles (both free iron oxide particles and silica nanoparticles containing iron oxide) were rinsed off after synthesis when large amounts of ethanol and water were used; thus, the fluorescence signals can only be obtained from the dye inside silica particles. So, the fluorescence observed in Figure 6(c) was from only silica particles containing the ruthenium dye. As shown in Figure 6(d), when the magnet was placed in the right corner of the image to cause the motion of the particles, all magnetic particles (iron oxide particles and magnetic silica particles containing 


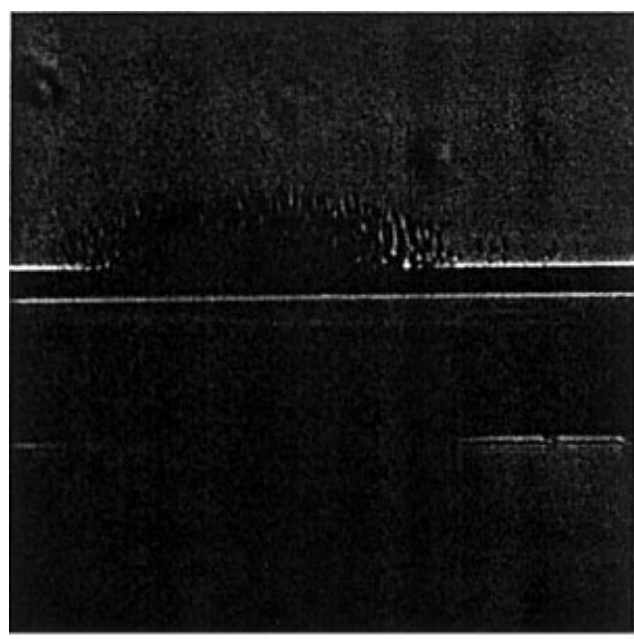

(a)

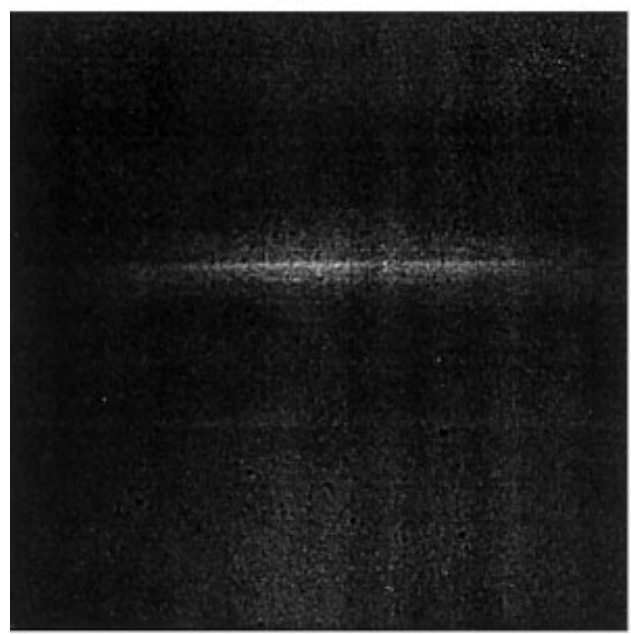

(c)

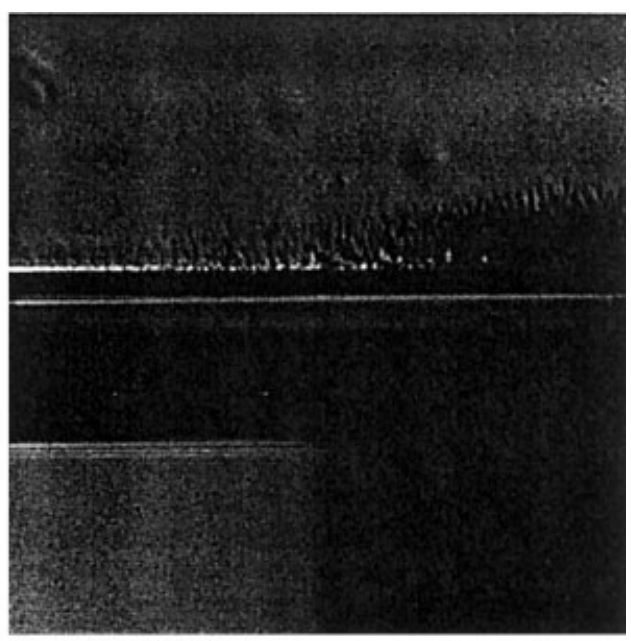

(b)

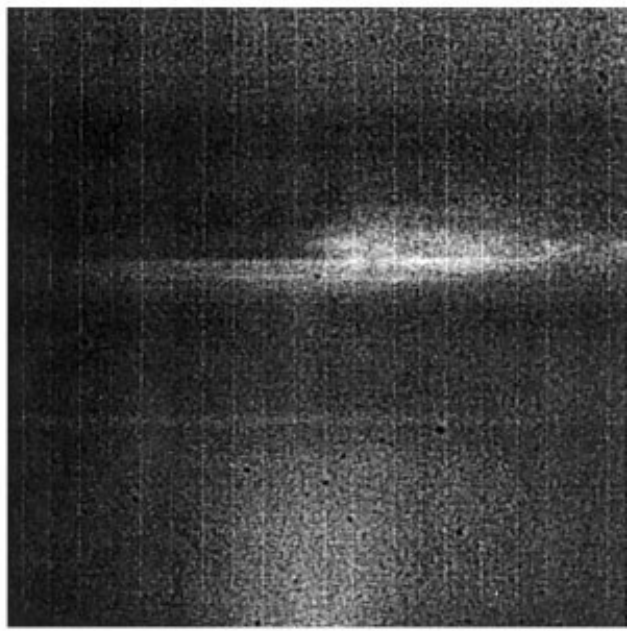

(d)

Figure 6. (a and b): Bright-field images of magnetic nanoparticles being pulled by the magnetic iron wire. The line in the middle of the image is a glass capillary. Images were taken after the sample was subjected to a magnetic field for (a) $0 \mathrm{~s}$ and (b) $14 \mathrm{~s}$. Exposure time: $200 \mathrm{~ms}$. (c and d): Fluorescence images of magnetic silica nanoparticles being pulled by the magnetic iron wire. The line in the middle of the image is a glass capillary. Images were taken after the sample was subjected to a magnetic field for (c) $0 \mathrm{~s}$ and (d) $2 \mathrm{~s}$. Exposure time: $1 \mathrm{~s}$.

iron oxide) were pulled toward the magnet [as in Fig. 6(a and b)], but only silica particles containing both the ruthenium dye and iron oxide contributed to the motion of the fluorescence signal. Thus, this experiment suggests the incorporation of iron oxide nanoparticles inside the silica particles. ${ }^{34}$ Furthermore, an example showing the encapsulation of iron oxide inside silica particles (prepared by a similar method) was previously reported by Lu et al., ${ }^{35}$ who examined the interface between iron oxide and silica particles using high-resolution transmission electron microscopy, which suggested the formation of a conformal coating of silica on the iron oxide nanoparticle core.

\section{SUMMARY AND CONCLUSIONS}

In this article, we have described a reproducible method for preparing PEG-coated silica nanoparticles at room temperature as a matrix for reagent-doped nanoparticles for in vivo applications inside intact biologic systems. The size of these particles can range from about $50-350 \mathrm{~nm}$ in diameter and were found by SEM and light-scattering experiments to be monodisperse. The chemical binding of PEG to the silica nanoparticles was suggested by infrared spectroscopy, and the PEG content on the silica nanoparticles was determined to be about $1-2 \%$. The PEG-coated silica nanoparticles showed enhanced colloidal stability when 
redispersed into aqueous $\mathrm{NaCl}$ solutions from the dried state because of the steric stabilization function of the PEG polymer grafted on the surface of particles. The nonspecific protein adsorption test indicated that the amount of protein adsorbed on the PEG-coated silica nanoparticle surface was remarkably reduced compared to that of the bare silica particles, which is critical for future in vivo applications of these particles. The encapsulation of magnetic components has also been demonstrated as one example of the possible applications of the PEG-coated silica nanoparticles. Future work will include the optimization of the PEG coating on particle surfaces and applications of these particles to in vivo studies.

Our thanks to Jeffery Anker for the help with the work on magnetic particle inclusion, and to the University of Michigan Electron Microbeam Analysis Laboratory for use of the SEM.

\section{References}

1. Santra S, Zhang P, Wang K, Tapec R, Tan W. Conjugation of biomolecules with luminophore-doped silica nanoparticles for photostable biomarkers. Anal Chem 2001;73:4988-4993.

2. Qhobosheane M, Santra S, Zhang P, Tan W. Biochemically functionalized silica nanoparticles. Analyst 2001;126:1274-1278.

3. Israelachvili J, Vigil G, Xu ZH, Steinberg S. Colloidal interactions of silica surfaces. Abstr Pap Am Chem Soc 1994;207:60 Phys Part 2.

4. Jain TK, Roy I, De TK, Maitra A. Nanometer silica particles encapsulating active compounds: A novel ceramic drug carrier. J Am Chem Soc 1998;120:11092-11095.

5. Tolnai G, Csempesz F, Kabai-Faix M, Kalman E, Keresztes Z, Kovacs AL, Ramsden JJ, Horvolgyi ZS. Preparation and characterization of surface-modified silica-nanoparticles. Langmuir 2001;17:2683-2687.

6. Shibata S, Taniguchi T, Yano T, Yamane M. Formation of watersoluble dye-doped silica particles. J Sol-Gel Sci Tech 1997;10: 263-268.

7. Van Helden AK, Jansen JW, Vrij A. Preparation and characterization of sperical monodisperse silica dispersions in nonaqueous solvents. J Colloid Interf Sci 1980;81:355-368.

8. Butterworth MD, Illum L, Davis SS. Preparation of ultrafine silica-and PEG-coated magnetite particles. Colloids Surf A 2001; 179:93-102.

9. Liu DM, Chen IW. Encapsulation of protein molecules in transparent porous silica matrices via an aqueous colloidal sol-gel process. Acta Mater 1999;47:4535-4544.

10. Chen SL. Preparation of monosize silica spheres and their crystalline stack. Colloids Surf A 1998;142:59-63.

11. Brinker JC, Scherer GW. Sol-gel science: The physics and chemistry of sol-gel processing. Boston: Academic Press; 1990.

12. Iler RK. The chemistry of silica. New York: John Wiley \& Sons; 1979.

13. Gruttner C, Teller J. New types of silica-fortified magnetic nanoparticles as tools for molecular biology applications. J Magn Magn Mater 1999;194:8-15.

14. Xu H, Aylott JW, Kopelman R, Miller TJ, Philbert MA. A real-time ratiometric method for the determination of molecular oxygen inside living cells using sol-gel based spherical optical nanosensors with applications to rat c6 glioma. Anal Chem 2001;73:4124-4133.

15. Clark HA, Kopelman R, Tjalkens R, Philbert MA. Optical nanosensors for chemical analysis inside single living cells: 2 . Sensors for ph and calcium and the intracellular application of pebble sensors. Anal Chem 1999;71:4837-4843.

16. Brasuel M, Kopelman R, Miller TJ, Tjalkens R, Philbert MA. Fluorescent nanosensors for intracellular chemical analysis: Decyl methacrylate liquid polymer matrix and ion-exchangebased potassium pebble sensors with real-time application to viable rat c6 glioma cells. Anal Chem 2001;73:2221-2228.

17. Quellec P, Gref R, Perrin L, Dellacherie E, Sommer F, Verbavatz JM, Alonso MJ. Protein encapsulation within polyethylene glycol-coated nanospheres: I. Physicochemical characterization. J Biomed Mater Res 1998;42:45-54.

18. Uhlmann DR, Teowee G, Boulton J. The future of sol-gel science and technology. J Sol-Gel Sci Tech 1997;8:1083-1091.

19. Dave BC, Dunn B, Valentine JS, Zink JI. Sol-gel encapsulation methods for biosensors. Anal Chem 1994;66:1120A-1127A.

20. Stöber W, Fink A, Bohn E. Controlled growth of monodisperse silica spheres in the micron size range. J Colloid Interf Sci 1968;26:62-69.

21. Bijsterbosch HD, Cohen Stuart MA, Fleer GJ. Effect of block and graft copolymers on the stability of colloidal silica. J Colloid Interf Sci 1999;210:37-42.

22. Vincent B. The preparation of colloidal particles having (postgrafted) terminally-attached polymer chains. Chem Eng Sci 1993;48:429-436.

23. Napper DH. Steric stabilization. J Colloid Interf Sci 1977;58: 390-407.

24. Kim KS, Cho SH, Shin JS. Preparation and characterization of monodisperse polyacrylamide microgels. Polym J 1995;27:508514.

25. Gref R, Luck M, Quellec P, Marchand M, Dellacherie Harnisch $\mathrm{S}$, Blunk T, Muller RH. 'Stealth' corona-core nanoparticles surface modified by polyethylene glycol (PEG): Influences of the corona (peg chain length and surface density) and of the core composition on phagocytic uptake and plasma protein adsorption. Colloids Surf B 2000;18:301-313.

26. Harris M. Poly(ethylene glycol) chemistry: Biotechnical and biomedical applications. New York: Plenum Press; 1992.

27. Ogris M, Brunner S, Schuller S, Kircheis R, Wagner E. PEGylated DNA/transferring-pei complexes: Reduced interaction with blood components, extended circulation in blood and potential for systemic gene delivery. Gene Ther 1999;6:595605.

28. Kingshott $\mathrm{P}$, Griessar HJ. Surfaces that resist bioadhesion. Curr Opin Solid St M 1999;4:403-412.

29. Alcantar NA, Aydil ES, Israelachvili JN. Polyethylene glycolcoated biocompatible surfaces. J Biomed Mater Res 2000;51: 343-351.

30. Lesot P, Chapuis S, Bayle JP, Rault J, Lafontaine E, Campero A, Judeinstein P. Structural-dynamical relationship in silica PEG hybrid gels. J Mater Chem 1998;8:147-151.

31. Matsuura H, Miyazawa T. Vibrational analysis of molten poly(ethylene glycol). J Polym Sci A 1969;7:1735.

32. Jeon SI, Lee JH, Andrade JD, Degennes PG. Protein surface interactions in the presence of polyethylene oxide: 1 . Simplified theory. J Colloid Interf Sci 1991;142:149-158.

33. Szleifer I. Protein adsorption on surfaces with grafted polymers: A theoretical approach. Biophys J 1997;72:595-612.

34. Yan F, Xu H, Anker J, Kopelman R, Ross B, Rehemtulla A, Reddy R. University of Michigan, 2002. Unpublished results.

35. Lu Y, Yin Y, Mayers BT, Xia Y. Modifying the surface properties of superparamagnetic iron oxide nanoparticles through a sol-gel approach. NANO Lett 2002;2:183-186. 